\title{
Philosophiques
}

\section{Controverse sur la causalité mentale dans l'action}

\section{Candida Jaci De Sousa Melo}

Volume 35, numéro 2, automne 2008

URI : https://id.erudit.org/iderudit/000433ar

DOI : https://doi.org/10.7202/000433ar

Aller au sommaire du numéro

\section{Éditeur(s)}

Société de philosophie du Québec

\section{ISSN}

0316-2923 (imprimé)

1492-1391 (numérique)

Découvrir la revue

\section{Citer cet article}

De Sousa Melo, C. J. (2008). Controverse sur la causalité mentale dans l'action. Philosophiques, 35(2), 345-367. https://doi.org/10.7202/000433ar

\section{Résumé de l'article}

Le problème métaphysique central en philosophie de l'esprit concerne la relation entre l'esprit et le corps des agents. Quand on tente d'expliquer, par exemple, le rapport entre les pensées et les actions humaines, on est alors immédiatement confronté avec la difficulté, apparemment insurmontable, d'expliquer la causalité mentale. On doit répondre à la question : nos états de pensée causent-ils effectivement ce que l'on fait? Bien sûr, nos croyances, nos intentions et nos désirs sont à la base de notre comportement, dira le sens commun et une longue tradition philosophique. Cependant, certains philosophes ont récemment manifesté des doutes à propos du rôle réel de nos pensées sur nos actions. Pour contrer ce scepticisme, il nous faut expliquer comment des états de pensée (considérés traditionnellement comme ontologiquement irréductibles aux états neuronaux) peuvent exercer leur rôle causal dans un monde fondamentalement physique. Je vais donner ici les raisons pour lesquelles je crois (comme beaucoup d'autres) que les états de pensée sont indispensables à l'explication complète de nos actions. Pour ce faire, je procéderai à une analyse approfondie de la relation entre la causalité mentale ou intentionnelle et la causalité physique. Je mettrai en perspective deux approches importantes sur la causalité mentale : celle de John Searle, qui défend la réalité des états mentaux et semble résister au réductionnisme, et celle de Jaegwon Kim, qui montre le péril auquel l'approche de Searle est exposée : la surdétermination causale! Selon Searle, le mental est un trait du monde physique (lequel est un système constitué de niveaux dont certains ont des traits mentaux). Aux yeux de Kim cette stratification en niveaux n'apporte aucune solution au problème de la causalité mentale. Kim a-t-il raison?
Ce document est protégé par la loi sur le droit d'auteur. L’utilisation des services d'Érudit (y compris la reproduction) est assujettie à sa politique d'utilisation que vous pouvez consulter en ligne.

https://apropos.erudit.org/fr/usagers/politique-dutilisation/ 


\title{
Controverse sur la causalité mentale dans l'action'
}

\author{
CANDIDA JACI DE SOUSA MELO \\ Université du Québec à Trois-Rivières \\ Candida_jaci@yahoo.com
}

\begin{abstract}
RÉSUMÉ. - Le problème métaphysique central en philosophie de l'esprit concerne la relation entre l'esprit et le corps des agents. Quand on tente d'expliquer, par exemple, le rapport entre les pensées et les actions humaines, on est alors immédiatement confronté avec la difficulté, apparemment insurmontable, d'expliquer la causalité mentale. On doit répondre à la question : nos états de pensée causent-ils effectivement ce que l'on fait? Bien sûr, nos croyances, nos intentions et nos désirs sont à la base de notre comportement, dira le sens commun et une longue tradition philosophique. Cependant, certains philosophes ont récemment manifesté des doutes à propos du rôle réel de nos pensées sur nos actions. Pour contrer ce scepticisme, il nous faut expliquer comment des états de pensée (considérés traditionnellement comme ontologiquement irréductibles aux états neuronaux) peuvent exercer leur rôle causal dans un monde fondamentalement physique. Je vais donner ici les raisons pour lesquelles je crois (comme beaucoup d'autres) que les états de pensée sont indispensables à l'explication complète de nos actions. Pour ce faire, je procéderai à une analyse approfondie de la relation entre la causalité mentale ou intentionnelle et la causalité physique. Je mettrai en perspective deux approches importantes sur la causalité mentale : celle de John Searle, qui défend la réalité des états mentaux et semble résister au réductionnisme, et celle de Jægwon Kim, qui montre le péril auquel l'approche de Searle est exposée : la surdétermination causale ! Selon Searle, le mental est un trait du monde physique (lequel est un système constitué de niveaux dont certains ont des traits mentaux). Aux yeux de Kim cette stratification en niveaux n'apporte aucune solution au problème de la causalité mentale. Kim a-t-il raison?
\end{abstract}

\begin{abstract}
The central metaphysical problem in philosophy of mind is concerned with the mind-body relation. When we try to explain relations between human thoughts and actions, we are immediately confronted with the difficulty of explaining the mental causation. We need to answer the question: do our thoughts effectively cause what we do ? Of course, according to common sense and a long philosophical tradition, our beliefs, intentions and desires are causes of our behaviour. But certain philosophers have recently expressed doubts about the real role of our thoughts in our actions. To avoid skepticism, we should explain how mental states (traditionally conceived as ontologically irreducible to neuronal
\end{abstract}

1. Cet article est le texte révisé de ma conférence au «Colloque international sur les attitudes et actions dans le discours » en hommage à Raymond Klibanski, tenu à Trois-Rivières lors du $75^{\mathrm{e}}$ Congrès de l'ACFAS, du 7 au 11 mai 2007. Je remercie D. Fisette, J.-L. Petit, D. Vanderveken et deux lecteurs anonymes de la revue Philosophiques pour leurs remarques critiques. 
states) can play any role in a world that is fundamentally physical. I will give here reasons why I believe like others that mental states are indispensable to the complete explanation of our actions. For this, I will analyse the relation between mental and physical causations. I will consider two important approaches on mental causation: the approach of John Searle who claims the reality of mental states and is opposed to reductionism, and the approach of Jaewon Kim who shows the difficulty to which Searle is exposed: causal overdetermination! According to Searle, mental properties are higher-level features in the physical world (a system orga-nised in levels). According to Kim, the stratification in levels does not give any solution to the mental causation problem. Is Kim right or not?

\section{Introduction}

De nombreux philosophes du courant analytique font actuellement des efforts considérables afin de déterminer si l'esprit joue réellement un rôle causal dans l'action humaine. Ils tentent de trouver une solution au problème de la causalité mentale. Il s'agit d'une extension du fameux problème de la relation entre la substance pensante (res cogitans) et la substance matérielle (res extensa) de Descartes. Dans son ontologie, Descartes distinguait les substances mentales qui pensent et dont les propriétés sont des modes de pensée, des substances matérielles qui sont étendues et dont les propriétés sont des modes d'extension. Dans son approche, les substances pensantes ne sont pas étendues et les substances étendues ne pensent pas. Donc, selon le dualisme cartésien, les traits de la pensée et ceux de l'extension s'excluent mutuellement. Descartes croyait cependant qu'il y avait quand même un lien entre les deux substances et que leur point de rencontre était l'épiphyse, qualifiée d' «organe de liaison ».

En philosophie de l'esprit, on ne considère plus le mental comme une substance immatérielle, métaphysiquement séparée de sa base physique (le corps/cerveau des agents). Les philosophes actuels qui défendent l'irréductibilité ontologique de l'esprit au corps se basent plutôt sur une autre forme de dualisme, celui des propriétés. Les partisans de ce nouveau courant défendent le monisme : la thèse selon laquelle, dans le monde il y a une seule substance, la substance physique. Cependant, cette substance peut avoir deux genres de propriétés: des propriétés mentales et des propriétés physiques - les premières étant irréductibles aux secondes. Cette approche pose néanmoins deux problèmes importants. Primo, si les propriétés mentales fonctionnent causalement et si on accepte le principe que le monde physique est causalement fermé, alors il y a surdétermination causale. Car si je lève intentionnellement mon index, cette action semble avoir deux causes : physique et mentale. Secundo, si les propriétés mentales n'exercent aucun rôle causal dans notre comportement, alors elles sont épiphénoménales. Dans ce cas, le mental doit être exclu des explications causales des actions. Donc, les partisans de l'irréductibilité du mental doivent répondre à la question: les propriétés mentales peuvent-elles figurer dans la structure des relations causales comme de véritables relata d'événements tels que les actions? 
J'aimerais présenter ce que j'appelle «le problème de la causalité mentale » en posant les questions suivantes: sommes-nous vraiment des agents capables de percevoir les objets et de représenter les faits du monde dans lequel nous vivons? Sommes-nous réellement des êtres conscients lorsque nous accomplissons intentionnellement des actions afin de changer le monde et d'interagir avec d'autres? Sommes-nous vraiment des êtres capables de réfléchir sur nos propres expériences : capables de les mémoriser et de se les remémorer lorsque nous en avons envie ? Sommes-nous réellement des êtres capables de raisonner sur la base de nos perceptions, croyances, intentions et désirs afin de décider et de délibérer sur les moyens à employer pour atteindre nos fins dans des circonstances totalement nouvelles ? À l'instar de la grande tradition philosophique, je pense que oui. Selon cette tradition, l'exercice de la pensée et l'action humaine sont fondamentalement caractérisés par la conscience (grâce à laquelle l'agent a une identité), par l'intentionnalité (grâce à laquelle il est dirigé vers les faits du monde) et par la rationalité (grâce à laquelle il fait des raisonnements théoriques et pratiques à partir de ses propres pensées). Pourtant, tenter d'expliquer pourquoi et comment un état conscient, intentionnel et rationnel peut causer un mouvement corporel (une action) reste une grande énigme philosophique.

On dit souvent que la source d'un problème philosophique est généralement liée à un conflit entre des hypothèses ou des croyances qu'on considère comme vraies. Il semble que le problème de la causalité mentale soit de cette sorte. D'une part, le sens commun et une longue tradition philosophique depuis les Grecs acceptent l'hypothèse que l'esprit fait une différence causale dans le monde. D'autre part, la tradition scientiste éminemment matérialiste et partisane du physicalisme ${ }^{2}$ argumente que le monde est causalement fermé. Voici comment Jaegwon Kim présente « la fermeture causale du monde physique »: "If a physical event has a cause at $\mathrm{t}$, then it has a physical cause at $\mathrm{t}^{3}$." Ce

2. Le physicalisme est une doctrine selon laquelle tout ce qui existe est physique (monisme). Comme Tim Crane l'a souligné dans son article "The Mental Causation Debate ", la motivation première du physicalisme était le besoin d'expliquer la place de la causalité mentale dans le monde physique. Les partisans de ce courant ont compris récemment que l'explication de la causalité mentale est leur plus grand problème. Certains d'entre eux tentent de le résoudre en défendant l'identité stricte entre le physique et le mental. Selon eux, si les propriétés mentales existent, elles sont alors identiques aux propriétés physiques. D'autres sont plus enclins à accepter l'existence du mental pour autant qu'il soit exhaustivement constitué ou composé d'éléments physiques. Dans cette optique, s'il y a des traits psychologiques dans le monde, ils sont fondamentalement constitués d'éléments physiques. D'autres comme Quine et les Churchland sont plutôt éliminativistes. Les articles de Tim Crane, "The Mental Causation Debate " in Proceedings of the Aristotelian Society Supplementary, volume LXIX, 1995, p. 211-236, et "Against Physicalism» in the Blackwell Companion to the Philosophy of Mind (édité par Samuel Guttenplan, Oxford, Blackwell, 1995, p. 479-489 sont très éclairants sur la question.

3. J. Kim, Physicalism, or Something Near Enough, 2005, Princeton University Press, p. 15. Ce principe est l'un des arguments les plus importants utilisés par les physicalistes contre la causalité mentale. Car si un événement physique a une cause, il a une cause physique suffisante. Les propriétés physiques sont alors causalement suffisantes pour leurs effets. Voir aussi l'article de D. Robb, «The Properties of Mental Causation » in Philosophical Quarterly 47, 1997, 178-95. 
principe véhicule l'idée fondamentale selon laquelle le domaine physique est autosuffisant (self-sufficient $)^{4}$ : on n'a pas besoin de sortir de ce domaine pour trouver la cause ou l'explication causale d'un événement physique, car tout ce qui se produit dans le monde physique est un effet (ou le résultat) d'une causalité physique antérieure et suffisante. Dans cette conception, tous les événements physiques peuvent être expliqués en termes de causalité physique, et ces explications établissent des conditions suffisantes pour produire l'événement qu'on tente d'expliquer.

Selon l'avis général, pareilles conditions rendent la défense de l'esprit très difficile. C'est pourquoi la clef de voûte de sa défense réside dans la causalité mentale. Il faut relever le défi de montrer l'indispensabilité de l'esprit dans le monde physique en expliquant par des arguments d'égale puissance pourquoi les propriétés mentales peuvent réellement causer d'autres événements.

\section{Du défi à surmonter}

Supposons que je désire écrire un poème, je perçois qu'il fait trop noir et je crois qu'en appuyant mon index sur l'interrupteur j'éclairerai ma chambre, alors je bouge volontairement mon corps afin de tenter d'appuyer mon index sur l'interrupteur de la lampe. On explique ainsi mon action en disant qu'elle est causée par des phénomènes mentaux tels que ma perception, mon désir, ma croyance, mon intention et ma tentative d'appuyer mon index sur l'interrupteur afin de changer l'état de choses du monde: passer de l'obscurité à la clarté. L'histoire causale de nos actions est ainsi, traditionnellement, expliquée en termes de causalité mentale ou intentionnelle ${ }^{5}$. Pareille explication est-elle correcte? Comment caractériser cette relation causale tout en respectant le principe de la fermeture causale du monde?

Le défi est de réussir à accommoder dans l'explication de nos actions (qui sont aussi des événements du monde physique) deux formes de causalité apparemment opposées : la causalité physique et la causalité mentale. Il faut mettre en évidence les différents traits constitutifs (physiques, logiques et psychologiques) de nos actions et expliquer pourquoi pareils traits sont, ensemble, leurs causes. Il faut le faire sans tomber dans le piège, apparemment inévitable, de la surdétermination causale. Ce problème, souligné par Kim, conduit directement à l'épiphénoménalisme.

4. J. Kim le dit explicitement dans son livre Physicalism, or Something Near Enough, op. cit. p. 16 (italique dans le texte).

5. Bien que le mouvement de mon corps soit à la base produit par des événements dont les causes sont physiques, les propriétés mentales comme la conscience, l'intentionnalité et la rationalité sont considérées dans la tradition comme des conditions nécessaires pour expliquer complètement mon comportement. Car pour bien déterminer ma façon d'agir, il faut bien expliquer pourquoi je suis dirigée vers une chose plutôt que vers une autre. Pourquoi je tente de lever mon bras jusqu'au mur où se trouve l'interrupteur plutôt que vers la fenêtre où il y a une plante? Comment répondre à pareille question sans considérer mes états de pensée, tels que ma perception spatiale des objets, mon désir de faire des choses, mon intention d'atteindre mon but et ma croyance d'en être capable? 


\section{Quelques distinctions philosophiques}

Afin de mieux préciser la nature des traits constitutifs des actions, j'aimerais introduire quelques distinctions philosophiques fondamentales concernant les notions traditionnelles liées au thème de la discussion. Il s'agit de clarifier les concepts clés de présentation, de représentation, d'état de choses, d'événement, de pensée et d'action.

Généralement, les théoriciens ${ }^{6}$ commencent l'étude de l'action en posant la question: "Pourquoi les actions humaines sont-elles différentes d'autres événements du monde naturel ? ». Pour répondre à pareille question, il faut tout d'abord admettre qu'aucun événement n'est une action s'il n'est accompli par un agent. Nous sommes des êtres qui pensent pour agir. En phénoménologie, en philosophie de l'esprit, en philosophie de l'action et du langage, le trait caractéristique central de la pensée est son intentionnalité ${ }^{7}$. Lorsque nous pensons à quelque chose, nous y pensons toujours sous certains aspects. En ce sens phénoménologique, l'intentionnalité est un phénomène mental dont la propriété principale est la directionnalité - le pouvoir propre à l'esprit d'être dirigé vers les faits du monde dont les choses font partie ${ }^{8}$. Ce qui est dirigé vers, ce qui est à propos de, ce qui présente ou représente quelque chose a alors par définition de l'intentionnalité. Dans la tradition philosophique depuis Descartes ${ }^{9}$, on distingue deux genres de pensées dirigées vers le monde : les pensées conceptuelles comme les croyances et les jugements, qui représentent les objets par des concepts, et les pensées non conceptuelles comme la perception, la mémoire et l'imagination, qui les présentent d'une façon plus directe et immédiate grâce aux sens ${ }^{10}$.

D'un point de vue ontologique, il y a différents types de faits dans le monde que nous pouvons expérimenter et penser. Certains sont appelés des états de choses, d'autres des événements. Parmi les états de choses, il y a les états purement physiques (la terre a une certaine masse et une forme, elle est

6. Voir par exemple Alvin Goldman, 1975, A Theory of Human Action, Princeton University Press; et Alfred R. Mele, 1997: The Philosophy of Action, Oxford University Press.

7. En général, l'étude de l'action humaine concerne plusieurs branches de la philosophie, dont la métaphysique, la phénoménologie, la philosophie de l'esprit, la philosophie du langage, etc. Dans tous ces domaines, la conscience, l'intentionnalité et la rationalité sont au cœur de l'explication des actions.

8. Wittgenstein a bien montré que «le monde est la totalité des faits, non des choses » dans son Tractatus logico-philosophique, 1922. Traduction anglaise de D. F. Pears and B. F. McGuiness, London: Routledge \& Kegan Paul, 1961. Ici j'utilise la traduction française de Gilles Gaston Granger, Paris: Gallimard, 1993. Voir $\mathbb{1} 1.1$.

9. Voir la "Sixième Méditation » in Descartes (1641), Méditations. Rééditées in Descartes, Euvres et lettres, textes présentés par A. Bridoux, Paris, Gallimard, Bibliothèque de la Pléiade, 1953.

10. Même si on perçoit des objets sous des concepts, la perception en tant que vécu d'expérience dépend de la présentation sensorielle des objets. Autrement, c'est une hallucination. Il faut souligner aussi que les traits des objets perçus sont distincts des traits de l'expérience consciente. 
une planète) qui existent, qu'on y pense ou non. Ces états de choses sont alors ontologiquement indépendants de nos sensations et de nos représentations mentales. Il y a en outre les états mentaux (comme les perceptions, les croyances, les désirs, les dépressions et les douleurs) qui sont ontologiquement dépendants de nos facultés subjectives. Parmi les états mentaux, il y a ceux appelés attitudes propositionnelles qui sont des états de pensée conceptuelle comme les croyances, les désirs, les intentions, lesquels sont pourvus d'un contenu représentationnel (généralement propositionnel). On perçoit des objets; on croit que des choses sont telles et telles; on désire qu'elles soient ainsi; on a parfois l'intention de faire en sorte qu'elles changent ${ }^{11}$, etc. Les autres états mentaux non conceptuels comme les angoisses, les dépressions et les anxiétés ne sont pas des pensées dirigées vers des faits ou des objets en particulier ${ }^{12}$.

À la différence des états de choses qui ont lieu, les événements surviennent et transforment le monde. D'un point de vue ontologique, il y a différents types d'événements. Certains sont purement physiques, leur occurrence dans le monde est totalement indépendante de nos pensées et actions comme le lever et le coucher du soleil, l'éruption d'un volcan et le mouvement des marées. D'autres, par contre, sont subjectifs, et leur occurrence dépend de nos pensées et actions. Tels sont par exemple : 1) les événements mentaux comme les actes de pensée conceptuelle (nos jugements, décisions, délibérations), et les actes de pensée non conceptuelle (nos perceptions visuelles, sonores, olfactives, auditives et tactiles, et nos mouvements corporels);2) les événements linguistiques comme les actes illocutoires (nos assertions, promesses, ordres, demandes, déclarations, salutations); 3 ) les événements sociaux comme les élections, les révolutions, les guerres et les mouvements de populations qui sont plutôt des processus ${ }^{13}$. Certains événements surviennent à un moment, la plupart durent pendant un intervalle de temps.

Ontologiquement, les états et les événements sont bien des faits. Parmi les événements il y a les actions individuelles et conjointes qui impliquent de l'intentionnalité humaine. Nous les accomplissons seuls ou à plusieurs. De même qu'il y a différents types d'événements, il y a différents types d'actions. Selon la tradition philosophique, à la base de toute action il y a des mouvements volontaires du corps ou plutôt des tentatives de pareils mouvements, comme bouger un membre, marcher et saisir un objet, qui sont des actions

11. Remarquons cependant que certains états de pensée comme l'amour, la haine, la crainte, etc. ont comme contenu un objet subsumé sous un concept (plutôt qu'une proposition). On aime, on hait et on craint certaines choses.

12. Il nous arrive parfois d'avoir certains états mentaux conscients (comme de l'anxiété) sans contenu bien déterminé. Pareils états sont dépourvus de contenu conceptuel ou représentationnel.

13. Voir à ce sujet, le $3^{\mathrm{e}}$ chapitre du livre de Terence Parsons, Events in The Semantics of English: A Study in Subatomic Semantics, Cambridge, MA, MIT Press, 1990. 
intentionnelles non conceptuelles et non verbales. Selon certains ${ }^{14}$, nous accomplissons pareilles actions en ayant à l'esprit une présentation (plutôt qu'une représentation) de leurs conditions de succès. Quand nous bougeons volontairement un membre ou un objet nous avons une présentation de la façon dont nous voulons qu'il soit. D'autres actions sont purement conceptuelles. Nous les accomplissons en nous représentant leurs conditions de succès. Parmi celles-ci, il y a les actes purement mentaux (comme juger, inférer, calculer, décider, tenter) et les actes de discours (comme affirmer, promettre, ordonner, déclarer, féliciter).

La philosophie de l'esprit traditionnelle distingue les états et les actes de pensée conceptuelle. Les croyances, les désirs, les intentions, les espoirs, les craintes, les peurs, les savoirs, les regrets, les tristesses et les joies que Descartes ${ }^{15}$ appelait passions de l'âme et que nous appelons aujourd'hui, suivant B. Russell ${ }^{16}$, des attitudes ne sont pas des actions que nous faisons, mais des états mentaux que nous avons. En revanche, les actes de pensée conceptuelle sont des actions intentionnelles (privées ou publiques) que les agents humains font consciemment: en pensant (ce sont des actes mentaux comme juger et s'engager) et en parlant (dans ce cas, ce sont des actes illocutoires selon la terminologie d'Austin ${ }^{17}$, comme déclarer et promettre). Ces états et actes de pensée conceptuelle sont les exemples paradigmatiques de phénomènes mentaux qui traduisent de l'intentionnalité. Pareils états et actes mentaux sont des pensées dont le contenu représente les objets ou les faits vers lesquels elles sont dirigées.

\section{Aperçu du débat entre deux approches philosophiques majeures}

\section{Le parti du réalisme mental}

En philosophie de l'esprit, il y a deux approches opposées concernant la nature ontologique des propriétés mentales. D'une part, certains penseurs soutiennent la réalité de l'esprit et tentent de le sauver de la réduction ontologique en invoquant ses caractéristiques propres et son influence dans certains événements physiques. D'autre part, certains philosophes envisagent de réduire l'esprit au physique ou même de l'éliminer des théories du comportement humain. Les partisans du réalisme mental soutiennent que la conscience, l'intentionnalité et la rationalité à l'œuvre lors de nos actions sont des propriétés mentales réelles

14. En particulier, John Searle dans son livre, Intentionality, Cambridge, Cambridge University Press, 1983.

15. R. Descartes, Les passions de l'âme (1649). Cet ouvrage a été réédité in Descartes: Euvres et lettres, textes présentés par A. Bridoux (1953). Op. cit.

16. B. Russell, An Inquiry into Meaning and Truth, George Allen and Unwin, London, 1976.

17. J. L. Austin, How to Do Things with Words, Oxford, Clarendon Press, 1962. 
des agents qui pensent. Or le test de l'authenticité (ou de la réalité) d'une propriété réside dans son efficacité causale. Les partisans de cette conception doivent alors montrer pourquoi l'intentionnalité, la conscience et la rationalité interviennent comme causes réelles de nos actions.

Dans ce courant, on accepte qu'il y ait une relation intime entre la causalité physique propre aux états neuronaux du cerveau des agents et la causalité mentale propre à leurs attitudes. Cela nous amène à croire que les événements mentaux sont produits par des événements physiques qui se déroulent ou se réalisent dans le corps/cerveau des agents. Ainsi, les états mentaux comme la douleur ou les attitudes comme les désirs sont causalement liés à des états cérébraux tels que des séquences d'enchaînements neuronaux. Une conséquence inévitable de ce principe est qu'il n'y aurait pas de propriétés mentales s'il n'y avait pas à la base des événements physiques (neuronaux) dans le corps/cerveau. Cependant, selon cette tradition, la seule stimulation corporelle ne suffisait pas à causer par exemple des actions volontaires. Donc, en dépit du fait qu'il y ait un certain consensus à propos de la connexion interne entre les propriétés mentales et les événements neuronaux, beaucoup s'opposent à l'idée de réduire les états de conscience, les états intentionnels et les états de raisons à des états purement neuronaux. Car pareille réduction mène inévitablement à l'épiphénoménalisme ${ }^{18}$.

\section{Le parti de l'irréalisme mental}

On peut raconter une autre histoire causale de nos actions, en suivant une autre tradition philosophique et scientifique qui se limite à la causalité physique, considérée comme totalement indépendante de la causalité mentale. Dans ce cas, on explique les actions humaines en faisant seulement référence aux processus physiques qui se déroulent dans le corps des agents, lesquels causent les mouvements corporels qui constituent les actions. Les adeptes de cette tradition présupposent que l'histoire causale des mouvements intentionnels du corps comme celui de lever mon index tient seulement à des activités neuronales, des neurotransmetteurs et des contractions musculaires, dont l'ontologie est objective, et qui sont totalement suffisantes pour l'expliquer.

18. Je remercie un évaluateur anonyme pour la précision historique à propos du premier philosophe à avoir étudié la thèse de l'épiphénoménalisme. En effet, selon Stanford Encyclopedia of Philosophy (http://plato.stanford.edu/entries), c'était le scientifique anglais Thomas H. Huxley (un des premiers à avoir adhéré à la théorie de l'évolution par la sélection naturelle de C. Darwin) qui a avancé la thèse que «les états physiques causent les états mentaux, mais ceux-ci ne causent rien " appelée "conscious automatism ", à la fin du dix-neuvième siècle. Cependant, selon The Cambridge Dictionary of Philosophy (Robert Audi, general editor, $2^{\mathrm{e}}$ édition, 1999, p. 685), William James fut le premier à avoir utilisé le terme "epiphenomena» en 1874 pour désigner des phénomènes qui manquent d'efficience causale. Selon la même source, James Ward forgea le terme «epiphenomenalism» en 1903. 
Les défenseurs de l'irréalisme mental ne s'engagent aucunement à reconnaître la réalité des phénomènes dont l'ontologie est subjective. Nombreux même sont ceux qui veulent la réduction du mental au physique. Dans ce cas, on identifie le mental aux processus neurobiologiques et, comme tel, il est dépourvu des traits qualitatifs phénoménaux que l'autre tradition considère comme irréductibles. À leurs yeux, il faut admettre la réduction si on veut éviter le problème de la surdétermination causale ${ }^{19}$. Certains d'entre eux, comme Dennett ${ }^{20}$, pensent qu'attribuer des états mentaux intentionnels aux dispositifs physiques n'est qu'une technique profitable, une stratégie utile qui facilite la compréhension de l'économie des systèmes. Car en moins de temps et en faisant moins d'efforts on comprend la description ou l'explication de leurs comportements. Mais attention! Assigner aux agents des états mentaux comme la douleur, ou des attitudes comme des croyances et des intentions sert juste à prédire et à décrire, mais aucunement à expliquer causalement leurs comportements. Selon cette optique instrumentaliste, on a besoin de la terminologie mentaliste aussi longtemps que l'avancement de la science ne permet pas de la remplacer.

Il y a d'ailleurs certains philosophes qui défendent une conception encore plus radicale. Selon les prévisions des Churchland ${ }^{21}$, de S. P. Stich ${ }^{22}$ et de S. Schiffer ${ }^{23}$, par exemple, toute trace mentale sera tôt ou tard éliminée dans les explications théoriques. Selon S. Schiffer, les événements mentaux sont des sous-espèces d'événements physiques, et les propriétés mentales seraient causalement superflues dans la théorie ${ }^{24}$. Dans cette optique éliminatrice, la psychologie du sens commun ou populaire, véhiculée par notre langage naturel, enchâssée dans les institutions sociales, sauvegardée par nos institutions légales et raffinée par les sciences comportementales et sociales est destinée à disparaître complètement de nos futures et véritables théories scientifiques $^{25}$.

19. Il faut souligner que cette manœuvre est trompeuse, car même si la réduction peut empêcher le péril de la surdétermination causale, elle ne peut éviter le danger de l'épiphénoménalisme souligné auparavant. Dans un cas comme dans l'autre, le mental est causalement impuissant.

20. Voir D. Dennett, 1987, The Intentional Stance, Cambridge, MA, The MIT Press.

21. P. S. Churchland, 1979, Scientific Realism and the Plasticity of Mind, Cambridge, Cambridge University Press. Et P. M. Churchland, 1986, Neurophilosophy, Cambridge, MA, The MIT Press.

22. S. P. Sitch, 1983, From Folk Psychology to Cognitive Science: The Case against Belief, Cambridge, MA, The MIT Press.

23. S. Schiffer, 1989, Remants of Meaning, Cambridge, The MIT Press.

24. S. Schiffer. Op. cit., ch. 6.

25. Si on prenait le temps d'évaluer le coût qu'implique une telle proposition, on serait vite convaincu qu'elle n'est pas une bonne affaire pour l'économie psychologique des agents humains. 


\section{Le naturalisme ou la stratégie de défense de la réalité du mental}

Pour contrer pareilles menaces de réduction ontologique ou d'élimination, certains philosophes dont Fodor ${ }^{26}$, Searle ${ }^{27}$ et Dretske ${ }^{28}$, partisans du réalisme mental, ont tenté de naturaliser l'esprit afin de lui donner un rôle causal. L'approche naturaliste de Searle, par exemple, tente de sauvegarder la place du mental dans les explications théoriques du comportement humain. Searle fait appel à la nature des propriétés mentales, à leur ontologie à la première personne (à leur subjectivité, leur qualité et leur aspect privé et phénoménal) pour faire valoir qu'elles ne se réduisent pas aux événements physiques de base. À ses yeux, pareils traits tout à fait particuliers sont des propriétés d'un niveau supérieur aux événements physiques (neuronaux) tout comme la solidité d'une table est un trait supérieur aux molécules qui la composent. Dans son livre, The Rediscovery of the Mind $^{29}$, Searle nous rappelle que l'irréductibilité des phénomènes mentaux est un argument classique, lequel repose sur les aspects subjectifs et phénoménaux (comme la qualité) qui sont d'ailleurs très bien défendus par d'autres que lui, comme Thomas Nagel ${ }^{30}$, Saul Kripke ${ }^{31}$, et Frank Jackson ${ }^{32}$.

26. Jerry Fodor, 1975, The Language of Thought, Cambridge, MA, Harvard University Press, et son article de 1990: "Making Mind Mater More» in A Theory of Content and Other Essays, Cambridge, MA, The MIT Press.

27. John R. Searle, 1983, Intentionality, Cambridge, Cambridge University Press. Et Searle, 1992, The Rediscovery of the Mind, Cambridge, MA, The MIT Press.

28. Fred Dretske, 1995, Naturalizing the Mind, Cambridge, MA, The MIT Press.

29. J. Searle, The Rediscovery of the Mind, Cambridge, Massachusetts, The MIT Press, 1992. Voir le chapitre 5, consacré à la question du "réductionnisme et l'irréductibilité de la conscience ", 111-126. Dans ce chapitre, voir, en particulier la section III : "Why Consciousness Is an Irreductible Feature of Physical Reality », p. 116-118

30. T. Nagel, dans son article "What Is It Like to Be a Bat?", in Philosophical Review, $\mathrm{n}^{\circ} 4$ LXXXIII, p. 435-450, montre le contraste entre l'objectivité des traits à la troisième personne (comme la dureté du fer) et la subjectivité des traits à la première personne (comme «l'effet que ça fait » d'avoir une douleur), mettant en évidence les traits subjectifs d'un état de conscience "l'effet que ça fait d'être...".

31. S. Kripke, dans son article "Naming and Necessity », in D. Davidson and G. Harman (ed.), Semantics of Natural Language, Dordrecht, Reidel, p. 253-355 et 763-769, fait une objection à la théorie de l'identité des états mentaux et des états neuronaux en présentant un argument modal. Selon lui, ce n'est pas une vérité nécessaire que l'état mental de douleur soit identique à la stimulation de la fibre-C du cerveau. Car il se peut qu'il y ait des cas où l'agent pourrait avoir ledit état cérébral sans avoir de la douleur et vice versa, souffrir d'une douleur sans avoir l'état cérébral correspondant. Cet argument exprime de façon très sophistiquée l'idée de base de l'objection du sens commun à la théorie de l'identité : on ne peut identifier quelque chose de mental à quelque chose de non mental, sans laisser tomber le mental.

32. F. Jackson, dans son article "Epiphenomenal Qualia ", in Philosophical Quarterly, 32, p. 127-136, contraste les deux types d'ontologies, l'objective et la subjective, en attirant l'attention sur le fait que la connaissance complète de la neurophysiologie d'un phénomène mental tel que la douleur n'est pas une condition suffisante pour que l'agent sache réellement ce qu'est la douleur. Car même s'il connaît parfaitement la description théorique du phénomène, il ignore néanmoins son aspect phénoménal : l'effet que ça fait d'avoir de la douleur. 
Searle explique sa propre approche en argumentant que les traits subjectifs des états mentaux sont des traits constitutifs (qui rendent possibles et constituent les modes) des vécus mentaux conscients. Voici un extrait de son argumentation :

Here is how it gœs: Consider what facts in the world make it the case that you are now in a certain conscious state such as pain. What fact in the world corresponds to your true statement, "I am now in pain" ? Naively, there seem to be at least two sorts of facts. First and more important, there is the fact that you are now having certain unpleasant conscious sensations, and you are experiencing these sensations from your subjective, first-person point of view. It is these sensations that are constitutive of your present pain. But the pain is also caused by certain underlying neurophysiological processes consisting in large part of patterns of neurons firing in your thalamus and other regions of your brain. Now suppose we tried to reduce the subjective, conscious, first-person sensation of pain to the objective, third-person patterns of neuron firings. Suppose we tried to say the pain is really "nothing but" the patterns of neuron firings. Well, if we tried such an ontological reduction, the essential features of the pain would be left out. No description of the third-person, objective, physiological facts would convey the subjective, first-person character of the pain, simply because the first-person features are different from the third-person features ${ }^{33}$.

En ce sens, les aspects subjectifs et phénoménaux, intrinsèques aux vécus mentaux, empêchent l'élimination de l'esprit et sa réduction à des propriétés non subjectives. Si les états mentaux ne peuvent être ni réduits ni éliminés, c'est qu'ils sont réels, et s'ils sont réels alors il n'y a pas de raison de les qualifier de propriétés immatérielles. Si les propriétés mentales sont réelles, alors elles interviennent dans notre comportement, elles ne sont donc pas épiphénoménales. Car si la solidité d'une table, par exemple, propriété réelle, causée par des événements physiques, n'est pas épiphénoménale, c'est qu'elle intervient causalement dans le comportement du système qui compose la table. (Elle affecte la trajectoire des molécules individuelles de l'ensemble des molécules qui constituent la table.) De même, l'intention d'appuyer l'index sur l'interrupteur, par exemple, n'est pas épiphénoménale parce qu'elle intervient dans le comportement de l'agent. Par exemple, elle oriente ses mouvements corporels, et cela affecte les molécules individuelles de l'ensemble des molécules qui constituent son corps.

Cependant, dans la citation Searle affirme: "But the pain is also caused by certain underlying neurophysiological processes consisting in large part of patterns of neurons firing in your thalamus and other regions of your brain." Quelles conséquences peut-on tirer de cette approche. Si les remarques de Kim sont justes, il y en a deux : 1) ou bien l'approche mène au problème de la surdétermination causale. Car il y a deux ensembles de causes indépendantes pour le même événement ; 2) ou bien l'approche est une forme de réduction. En effet,

33. J. Searle, The Rediscovery of the Mind. Op. cit., p. 117. 
si la causalité physique des micro-éléments est suffisante pour causer le processus à tous les niveaux, alors la causalité mentale n'est qu'un effet. Il y a dépendance et donc place à la réduction. Mais quelle forme de réduction Searle adopte-t-il : s'agit-il d'une réduction ontologique, logique ou épistémologique?

\section{Le problème de la surdétermination causale}

Supposons que les prévisions avancées par la conception éliminatrice soient fausses; que ses adeptes ont tort de croire que la psychologie populaire est totalement incorrecte et qu'elle va définitivement disparaître! Il reste encore une menace de réduction, car il y a deux histoires causales en compétition: l'une mentale et l'autre physique. Il y a alors selon $\mathrm{Kim}^{34}$ un problème de surdétermination causale. Quand un effet est produit par deux causes indépendantes et qu'une seule d'entre elles aurait suffi, on dit qu'il y a surdétermination causale $^{35}$. Selon Kim, ce problème crée des difficultés à deux niveaux : le premier niveau concerne la nature des événements considérés. Cette première difficulté touche surtout ceux qui soutiennent encore que les états mentaux sont des événements immatériels. Le second niveau concerne la nature des propriétés considérées. La seconde difficulté touche surtout ceux qui soutiennent que les propriétés mentales sont irréductibles aux propriétés physiques, non parce qu'elles sont immatérielles, mais parce qu'elles sont intrinsèquement subjectives. Bien entendu, le problème de la surdétermination causale ne concerne pas l'irréalisme mental mais l'autre camp, à cause de l'incompatibilité entre les quatre propositions suivantes : 1) l'hypothèse de l'irréductibilité ontologique de l'esprit: les états mentaux sont à la fois réels et irréductibles aux états physiques. 2) L'hypothèse de la causalité mentale: les états mentaux causent d'autres événements physiques. 3) Le principe de fermeture causale du monde physique: tout événement physique a des causes physiques suffisantes. 4) L'hypothèse du naturalisme biologique: il n'y a pas de surdétermination causale. Lorsqu'on met ensemble, par exemple, les propositions 1, 2 et 3 :

1) Le désir de lever mon index, par exemple, est un état réel du monde, irréductible à l'état neuronal qui le cause.

2) C'est le désir de lever mon index, par exemple, qui cause son mouvement ou en est responsable.

3) La levée de mon index a des causes physiques suffisantes. Autrement, il se retrouverait en l'air par une cause mystérieuse ou miraculeuse.

il y a alors surdétermination causale! Le désir et l'état neuronal d'où il émerge causent chacun mon action intentionnelle de lever mon index.

34. J. Kim, Mind in a Physical World: An Essay on the Mind-Body Problem and Mental Causation. A Bradford Book, Cambridge, MA, MIT Press, 1998.

35. Voir aussi Robert Audi, The Cambridge Dictionary of Philosophy, éd. Général, deuxième édition, 1999, p. 127. 


\section{Comment certaines approches surmontent-elles cette difficulté}

Certains tentent de surmonter la difficulté de la surdétermination causale en disant que les événements mentaux sont des traits du système cérébral. C'est le cas de Searle dont l'approche soutient que: "All of our conscious states are caused by lower-level neuronal process in the brain, and they are themselves features of the brain ${ }^{36}$.» À ses yeux, ils font partie du monde: "As parts of the real world, consciousness, intentionality and rationality are "physical" phenomena, like anything else ${ }^{37}$. » Searle écarte ainsi le problème de la dichotomie ontologique entre le mental et le physique, qu'il accuse d'être un héritage cartésien, en disant qu'il s'agit d'événements hybrides à la fois physiques et mentaux. Selon lui : "Le fait qu'un trait est mental n'implique pas qu'il n'est pas physique; le fait qu'un trait est physique n'implique pas qu'il n'est pas mental $^{38}$. " Searle considère comme un fait objectif que le monde physique contient des systèmes comme le cerveau, qui a des états psychologiques, et comme un fait physique que de tels systèmes aient des traits mentaux ${ }^{39}$. Son programme naturaliste semble donc défendre à la fois la réalité ontologique des propriétés mentales, en tant que traits irréductibles à des activités neuronales, et la réduction, quant à la recherche de propriétés causales sous-jacentes qui, elles, ne doivent pas être mentales ${ }^{40}$.

L'inconvénient avec cette approche est qu'elle semble tomber dans le piège de l'épiphénoménalisme. Car lorsqu'on met ensemble les propositions 2, 3, et 4 :

2) La levée de mon index est causée par mon désir d'appuyer sur l'interrupteur, par exemple;

3) Tout événement physique (une action, par exemple) a des causes suffisantes au niveau sous-jacent;

4) Il n'y a pas de surdétermination causale.

alors, la proposition 2 est fausse! Mon désir n'est qu'un épiphénomène.

Si les propriétés mentales sont fondées, réalisées et causalement dépendantes des structures neurobiologiques (sans doute physiques), alors il semble que nos états de conscience ne soient pas autonomes. En tout cas, ils sont inefficaces pour causer tout seuls des actions intentionnelles. Ainsi, d'une part, Searle est convaincu que les propriétés mentales existent à un certain niveau; d'autre part, son naturalisme échoue à déterminer pourquoi pareilles propriétés ne sont

36. Ibidem, p. 272. Il soutient cela tout d'abord dans son ouvrage de 1983, Intentionaliy: An Essay in the Philosophy of Mind. Op. cit. Et ensuite dans son ouvrage de 1992: The Rediscovery of the Mind. Op. cit.

37. J. Searle, Rationality in Action. Cambridge, MA, The MIT Press, 2001, ch. 9, p. 270. Entre guillemets dans le texte.

38. J. Searle, The Rediscovery of the Mind, Op. cit., p. 14.

39. J. Searle, Intentionaliy. Op. cit., p. ix.

40. On verra plus loin que l'approche de Searle, généralement considérée comme une forme de dualisme de propriété, n'en est pas une. 


\section{Philosophiques / Automne 2008}

pas des relatas dans les relations causales des événements intentionnels. Le problème de la surdétermination causale apparaît alors comme étant plus lié à des critères épistémiques et moins à la nature ontologique des propriétés en dispute. Il s'agit de vérifier si l'approche de la description de leurs traits distinctifs basée sur la distinction en niveaux n'entraîne pas une surdétermination causale au niveau métaphysique.

\section{L'approche de la distinction en niveaux sauve-t-elle la causalité mentale ?}

Surmonter le problème de la causalité mentale concerne la difficulté de montrer que les propriétés mentales sont irréductibles aux propriétés physiques et qu'elles ne sont pas épiphénoménales. Surmonter pareilles contraintes est d'une importance capitale pour l'approche de Searle. Pour y parvenir, il adopte la conception selon laquelle la réalité est organisée en plusieurs paliers ou niveaux, ce que Kim appelle en anglais "levelism ${ }^{41}$ ", dans ma traduction la « distinction en niveaux». Selon Searle :

Le même système peut être décrit à différents niveaux... Et le point important apporté par cette "distinction en niveaux " est de reconnaitre comme causalement réels les niveaux de description où, dans certains cas au moins, les niveaux supérieurs sont à la fois causés par le comportement des éléments au niveau inférieur et réalisés dans le système composé de pareils éléments ${ }^{42}$.

Plus particulièrement, le comportement des éléments du niveau micro, qu'on présume être des neurones et des synapses, cause le niveau macro, qu'on présume être le système des propriétés ou traits de la conscience et de l'intentionnalité ${ }^{43}$. Comme Searle le dit, cette forme de causalité est commune dans la nature. Par exemple, la liquidité de l'eau, propriété macro, est causalement expliquée par le comportement des éléments au niveau micro, les molécules roulant les unes sur les autres de façon aléatoire. La liquidité est un trait réel du monde, une propriété émergente du système des molécules; elle n'est aucunement inerte ou épiphénoménale. Mais la liquidité n'est pas un événement subjectif. Elle est entièrement déterminée par le comportement des micro-éléments qui la compose. Que se passe-t-il dans le cas de la conscience, qui est un phénomène subjectif?

41. Selon Jægwon Kim, Mind in a Physical World, Cambridge, MA, MIT Press, 1998, le « levelisme « est un modèle qui voit le monde stratifié en différents niveaux organisés dans une structure hiérarchique. Ce modèle a remplacé la dichotomie de Descartes. Voir p. 15.

42. J. R. Searle, The Construction of Social Reality, New York, London, The Free Press, 1985. Voir p. 219.

43. Kim développe une critique de l'approche de Searle, et son argument montre que la causalité entre différents niveaux n'élimine pas le problème de la surdétermination. Voir son livre: Mind in a Physical World. Op. cit. p. 47-49. 


\section{L'argument de Kim contre l'approche de Searle}

Selon Kim, cette approche n'élimine pas la causalité mentale. Car l'indépendance de deux causes contredit le principe physique de la fermeture causale du monde. Voici l'argumentation de Kim:

Selon Searle, on peut alors affirmer, sans peur de surdétermination, toutes les choses suivantes:

1) que $M$ (la douleur) cause $M^{*}$ (le désir de prendre une aspirine);

2) que $P$ (une séquence neuronale) cause $P *$ (une autre séquence neuronale);

3) $\mathrm{P}$ cause $\mathrm{M}$; et

4) $P^{*}$ cause $M^{*}$.

Cela est parfaitement correct, nous rassure Searle, parce que 1 et 2 sont des «descriptions du même système données à différents niveaux ». C'est certainement un point de vue qu'on peut soutenir; cependant, il n'est pas du tout sûr que Searle puisse ou veuille affirmer cela. Car en disant que « la douleur cause un désir de prendre l'aspirine» et que «les séquences neuronales $\mathrm{P}$ causent les séquences neuronales $\mathrm{P}^{*}$ sont des descriptions de la même situation est plausible seulement si l'on est prêt à dire que "douleur » et " séquences neuronales » sont des descriptions du même phénomène (à "différents niveaux"). Il en va de même pour l'autre paire «le désir de prendre l'aspirine " et «les séquences neuronales $\mathrm{P} *$ ». C'est plausible seulement si l'on est prêt à dire que la douleur est égale aux séquences neuronales $P$, et que le désir d'aspirine est égal aux séquences neuronales $\mathrm{P}^{*}$. En ce qui me concerne, je ne pense pas que cela soit un mouvement implausible; en fait, certaines formes de réductionnismes de ce type peuvent devenir la seule conception viable si les événements mentaux doivent réellement avoir un rôle causal dans le monde physique. Mais retournons à Searle : il doit être clair que l'identité psychoneuronale de ce genre met en péril l'approche de Searle selon laquelle les séquences neuronales $\mathrm{P}$ causent la douleur, et les séquences neuronales $\mathrm{P}^{*}$ causent le désir de prendre de l'aspirine. Searle, naturellement, veut dire que pareilles descriptions sont à des niveaux différents. Mais qu'est-ce que cela signifie ? Et quelle différence cela fait-il ? ${ }^{44}$

Dans son argumentation, Kim soulève la difficulté de la thèse de l'irréductibilité des propriétés mentales, le déséquilibre métaphysique de ce dualisme. En effet, si les propriétés mentales surviennent sur les propriétés physiques (la relation de réalisation est une relation survenante), alors les propriétés mentales, en tant que propriétés mentales, pourraient conférer des pouvoirs causaux à leurs instances. Or la clôture causale du domaine physique se charge déjà de préétablir ces propriétés survenantes.

Searle est d'accord avec Kim sur un point. Il dit explicitement :

La conscience est réductible aux processus du cerveau, car tous les traits de la conscience sont considérés comme étant biologiquement causés par des processus

44. J. Kim, Mind in a Physical World, op. cit., p. 49-50. Les italiques et soulignements sont de moi. 
qui se déroulent dans le cerveau, et la conscience n'a pas de pouvoir causal qui s'ajoute au pouvoir causal de la neurobiologie sous-jacente ${ }^{45}$.

Cette thèse est en accord avec son approche et en est même une conséquence, laquelle approche est greffée sur une image métaphysique du monde hiérarchisée par niveaux et ne contenant qu'une seule substance, le physique. Mais si les propriétés mentales n'exercent aucune pression causale dans le monde physique, on voit mal comment on pourrait les faire exister réellement. À cela Searle objecte à Kim :

$[\ldots]$ in the case of consciousness, causal reducibility does not lead to ontological reducibility. From the fact that consciousness is entirely accounted for causally by neuron firings, for example, it does not follow that consciousness is nothing but neuron firings. Why not? What is the difference between consciousness and other phenomena that undergo an ontological reduction on the basis of a causal reduction, phenomena such as colour and solidity? The difference is that consciousness has first-person ontology; that is, it only exists as experienced by some human or animal, and therefore, it cannot be reduced to something that has third-person ontology, something that exists independently of experiences. It is as simple as that ${ }^{46}$.

S'il y a un niveau de description où les processus du cerveau sont constitués de séquences réelles et d'irréductibles états de conscience, et s'il y a un autre niveau de description où les processus du cerveau sont constitués de phénomènes purement biologiques, et si les états de conscience ne sont pas ontologiquement réductibles aux phénomènes neurobiologiques, «[...] alors ces deux niveaux doivent exister séparément ». Qu'en est-il alors de l'irréductibilité de telles propriétés ${ }^{47}$ Searle souligne que si la réalité des propriétés mentales est irréductible, cela n'implique pas qu'il y ait des types d'entités ou des types de propriétés qui s'ajouteraient au système du cerveau dans lequel elles se réalisent. La conscience dans le cerveau n'est donc pas une entité ou une propriété séparée du système. Elle est juste l'état du cerveau. Voilà ce qu'il veut dire quand il affirme :

Le même système admet différentes descriptions causales à différents niveaux et cela n'implique pas la surdétermination causale ou la faille de la fermeture

45. J. R. Searle, 2002, "Why I Am Not a Property Dualist ", in Journal of Consciousness Studies, v. 9 , n ${ }^{\circ} 12,2002$, p. 57-64

46. Searle: "Why I Am Not a Property Dualist ", op. cit. p. 60.

47. Dans cet article, "Why I Am Not a Property Dualist ", op. cit., Searle parle seulement du phénomène de la conscience. Mais on peut déduire que son approche s'applique aussi à l'intentionnalité, car il dit plus loin, dans la même page: "I think that the conclusions of the discussion will extend to other features of the mind-body problem, such as, for example, the relationship between intentionality and brain process [...]». Voir p. 57. 
causale du monde. Qui plus est, rien de tout ça n'implique aucunement que la chaleur, la liquidité et la solidité soient épiphénoménales ${ }^{48}$.

Ce qu'il entend par là, c'est que son approche ne postule pas deux ensembles de causes différentes, la conscience et les neurones, mais seulement un ensemble unique, décrit à différents niveaux. Car la conscience n'est rien d'autre que l'état dans lequel se trouve le système des neurones, de la même façon que la solidité n'est rien d'autre que l'état dans lequel se trouve le système de molécules. En outre, il explique pourquoi sa façon d'expliquer la causalité mentale en la réduisant en quelque sorte aux structures neurobiologiques ne rend pas l'esprit épiphénoménal. À ses yeux, le simple fait qu'une caractéristique supérieure ou systémique soit fixée par des micro-éléments n'établit pas que la caractéristique systémique est épiphénoménale. Bien au contraire, même si la conscience est fixée par le comportement neuronal, elle n'est pas pour autant épiphénoménale, car on agit parfois sur la base de processus conscients (de là les processus neuronaux) par le biais desquels le moi ou l'ego rationnel prend des décisions en se fondant sur des raisons ${ }^{49}$.

Searle peut-il refuser la thèse que le mental est épiphénoménal et soutenir qu'il est causalement réductible aux processus neuronaux? Qu'est-ce que cela implique pour la thèse de la liberté dans l'action?

Dans Intentionality, Searle développe une approche selon laquelle la causalité intentionnelle est un processus réglé par la transitivité. Selon lui, lorsqu'un agent exécute une intention préalable, cette intention préalable cause une intention en action qui cause elle-même l'action intentionnelle :

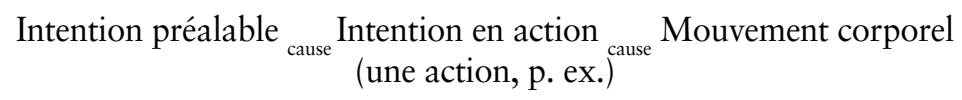

48. J. R. Searle, "Consciousness, the Brain and the Connection Principle: A reply ». In Philosophy and Phenomenological Research, vol. LV, $\mathrm{n}^{\circ}$ 1, mars 1995, p. 217-232. Voir la page 219.

49. Dans son livre, Liberté et neurobiologie, édité et traduit en français par Patrick Savidan, paru à Paris chez Bernard Grasset, 2004, Searle parle des conditions qu'un système (le cerveau) doit satisfaire pour avoir des états mentaux conscients. Premièrement, la conscience telle qu'elle est causée par des processus neuronaux et telle qu'elle se réalise dans un système neuronal a un fonctionnement causal vis-à-vis du corps. Deuxièmement, le cerveau cause et soutient l'existence d'un moi conscient capable de prendre des décisions rationnelles et de les traduire en actions. Il ne suffit pas que la conscience ait des effets physiques sur le corps. Autrement dit, il ne suffit pas d'avoir une description neurobiologique de la causalité mentale, il faut avoir une description du moi rationnel et volitif. Comment le cerveau crée-t-il un moi ? Les éléments qu'un organisme demande pour avoir un moi sont: a) un champ unifié de conscience; b) la capacité de délibérer sur la base de raisons - cette capacité n'implique pas seulement des capacités cognitives de perception et de mémoire, mais aussi la capacité de coordonner des états intentionnels, afin de parvenir à des états de décisions rationnelles; c) l'organisme doit être capable d'accomplir des actions. Voir les pages 62-66. 
Selon ce schéma, il y a un lien causal entre les pensées des agents et leurs actions. En outre, Searle explique que la transitivité n'implique pas le déterminisme. Chaque action intentionnelle est, selon lui, consciente et apparemment libre. Mais son approche défend aussi que, pour la même séquence causale ${ }^{50}$, il y a corrélation entre les deux niveaux de descriptions :

$$
\begin{gathered}
\text { Intentions préalables }{ }_{\text {cause }}^{\text {Intention en action }} \text { (une action, p. ex.) } \\
\mathrm{t} \text { (cause } \& \text { réalise) } \mathrm{t} \text { (cause } \& \text { réalise) t (cause } \& \text { réalise) }
\end{gathered}
$$

Neurones activés ${ }_{\text {causent }}$ Changement physiologique ${ }_{\text {cause }}$ Changement physiologique

Or ce schéma représente une relation de causalité totalement déterministe. L'intention en action, placée au niveau supérieur, cause le mouvement corporel, alors qu'au niveau inférieur l'activité des neurones cause des changements neurophysiologiques. Comme à chaque point de la séquence, le niveau inférieur cause et réalise le niveau supérieur, les changements neurobiologiques causent les changements psychologiques. Que se passe-t-il alors quand, après avoir délibéré, on change d'avis, quand on décide, par exemple, de ne rien faire ou d'agir différemment ? Puisque le système est entièrement déterminé causalement au niveau inférieur (neuronal) qu'en est-il de notre sentiment de liberté ? Est-il réel ou illusoire?

Dans son livre plus récent, Rationality in Action ${ }^{51}$, Searle admet que dans le cas de la cognition, le mode passif de conscience propre à la perception et à la mémoire, il y a une parfaite symétrie entre les états cognitifs au niveau psychologique et les états neuronaux au niveau neurophysiologique. À n'importe quel point du système, les traits des micro-éléments sont causalement suffisants pour déterminer l'état de conscience à ce point-là. Il y a déterminisme neurobiologique dans le cas cognitif. Par contre, dans le cas de la volition, il semble y avoir, lors de la décision, un «écart » entre les états mentaux, entre ce qui se passe alors et ce qui se passera plus tard. Nous pourrions prendre une autre décision et, une fois la décision prise, ne pas exécuter cette décision. Y a-t-il un pareil écart au niveau neurobiologique? Selon Searle, deux hypothèses sont envisageables: 1 ) ou bien l'indéterminisme psychologique n'est pas réalisé au niveau neurophysiologique et il y a un écart mental mais pas d'écart neurophysiologique, et toute expérience de liberté est alors un épiphénomène ; 2 ) ou bien il y a une symétrie parfaite entre les deux niveaux de description, à la fois indéterminisme psychologique et neurophysiologique, et l'écart mental est un signe de liberté réelle.

50. J. Searle, Intentionality, op. cit., p. 94. Si on agit sur la base de nos intentions et désirs, c'est qu'ils fonctionnent causalement, mais cela n'implique pas qu'on ne pourrait pas agir autrement. Il n'est donc pas vrai que lorsqu'on a décidé de faire quelque chose (sur la base de nos désirs et intentions) on va nécessairement l'exécuter.

51. J. Searle, Rationality in Action, op. cit., p. 283-292. 
La première hypothèse (illustrée par le schéma ci-dessous), présuppose qu'il y a indéterminisme psychologique et déterminisme neurophysiologique. L'inconvénient majeur de cette hypothèse est qu'elle mène à l'épiphénoménalisme.
Délibération de faire $\mathrm{A}_{\text {cause avec écart }}$ Décision de faire $\mathrm{A}_{\text {cause avec écart }}$ Réalisation de A $\mathrm{t}$ (cause $\&$ réalise) t(cause $\&$ réalise) t(cause $\&$ réalise)

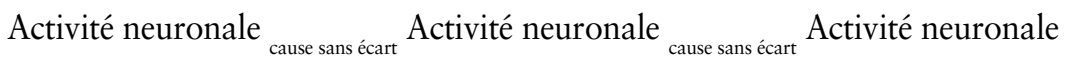

Selon ce schéma, il y a une asymétrie entre les deux niveaux de descriptions. Bien qu'on éprouve mentalement un écart dans la délibération entre les raisons de faire une action et la décision de la faire à l'issue de la délibération, il n'y a pas d'écart au niveau neurophysiologique entre causes et réalisations neuronales des actions mentales. Quelles peuvent être les conséquences en ce qui concerne la question de la causalité mentale ? Bien entendu, notre sentiment de contrôle sur le processus de délibération est réel, nous avons bel et bien l'expérience de prendre librement notre décision à l'issue de la délibération et de ne pas être forcés d'exécuter nos intentions préalables. Cependant, bien que nos expériences conscientes de liberté soient des faits réels, causées par des écarts au niveau mental ${ }^{52}$, elles sont épiphénoménales, car le système est entièrement déterministe au niveau inférieur. Selon Searle, cette hypothèse possible est néanmoins très improbable, car elle est contraire à nos idées mêmes sur l'évolution:

It would have the consequence that the incredible elaborate, complex, sensitive, and - above all - biologically expensive system of human and animal conscious rational decision making would actually make no difference whatever to the life and survival of the organisms ${ }^{53}$.

La seconde hypothèse (illustrée par le schéma suivant), présuppose une symétrie parfaite entre les deux niveaux. L'indéterminisme existe à la fois au niveau psychologique et au niveau neurophysiologique:
Délibération de faire $\mathrm{A}_{\text {cause avec écart }}$ Décision de faire $\mathrm{A}_{\text {cause avec écart }}$ Réalisation de A t (cause $\&$ réalise) t (cause $\&$ réalise) t(cause $\&$ réalise)

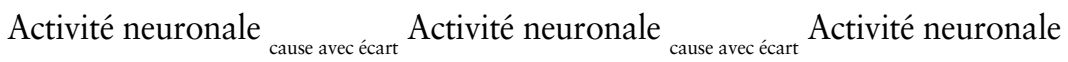

Ce schéma représente une relation de causalité indéterministe. L'activité neuronale manque de conditions causalement suffisantes pour causer les

52. La raison des écarts est due à la durée du temps nécessaire d'abord pour raisonner, ensuite pour décider et enfin pour accomplir l'action. Ici, la question concerne davantage les conditions nécessaires et suffisantes du mental pour causer les événements qui s'en suivent jusqu'à la fin du processus. Parfois on prend du temps pour délibérer, pour exécuter ses décisions, et parfois on renonce à agir comme prévu.

53. J. Searle, Rationality and Action, op. cit., p. 286. 
changements psychologiques. Qu'est-ce que cela implique ? Primo, un certain holisme : la conscience est un système de traits, c'est le trait d'un système complet, elle est présente dans tous les endroits importants du système. Cela veut dire que l'ensemble du système bouge d'une façon causale sans être déterminé par des conditions causalement suffisantes. Le système bouge entièrement pendant la délibération, vers la prise de décision, vers l'exécution de la décision sous forme d'actions. Comment, du point de vue neurobiologique, l'écart fonctionne-t-il pour ce qui est de l'agir ? Si la seconde hypothèse prévaut, alors on doit présupposer que la conscience du système a des effets causaux sur les éléments qui le composent. De même que la liquidité de l'eau a des effets sur les molécules, bien que l'eau soit composée de molécules, ainsi la conscience a des effets sur les neurones et synapses, même si le système de conscience est formé de ces éléments individuels.

Mais cette analogie entre le système causal de l'eau et le système causal de la conscience arrête de fonctionner à ce point précis : le comportement des molécules d'eau est entièrement déterminé alors que, selon la seconde hypothèse, le comportement du cerveau conscient ne l'est pas. La question qui se pose ici est de savoir comment la neurobiologie pourrait travailler sur la base de cette hypothèse. Searle ne répond pas à la question. Selon lui, les recherches menées actuellement en sciences neurobiologiques et cognitives pourront peut-être démontrer comment la liberté d'agir affecte la structure complète du système.

\section{Conclusion}

Même après ces clarifications, on est porté à croire que le "naturalisme biologique » de Searle l'engage quand même à un certain type de dualisme. Car s'il admet que la causalité mentale est liée aux structures physiques, il refuse de réduire les propriétés mentales à tout ce qui reviendrait à une ontologie à la troisième personne. Comment alors concilier cette position avec celle exposée dans son article: «Why I Am Not a Property Dualist ${ }^{54}$ », laquelle refuse le dualisme des propriétés accusé d'être une conception erronée ? À ses yeux, la question de savoir s'il y a un, deux ou trois types de propriétés métaphysiques fondamentales n'a aucun sens. Car il n'y a qu'un seul monde, le monde physique, qui a plusieurs types de traits différents, comme les traits biologiques et les traits psychologiques, lesquels sont bien des phénomènes biologiques reliés au système neuronal, mais leurs caractéristiques intrinsèques sont particulièrement différentes des autres traits du système. C'est pourquoi il y a réduction causale mais pas de réduction ontologique.

D'une part, Kim a raison d'accuser l'approche de Searle d'être une forme de réductionnisme. Car Searle admet malgré tout que certaines caractéristiques d'un système cérébral comme les états de conscience, l'intentionnalité et

54. J. Searle, 2002, «Why I Am Not a Property Dualist », Journal of Consciousness Studies, 9, $\mathrm{n}^{\circ} 12,2002$, op. cit. p. 57-64. 
les états rationnels sont juste des traits qui surviennent lorsque le système est activé par certains stimulus. Ils ne sont pas épiphénoménaux; les états volitifs, par exemple, peuvent affecter l'activité neuronale en causant des écarts lors des séquences d'événements intentionnels et rationnels comme la délibération, la prise de décision et l'accomplissement de l'action. Cependant, et cela est important, le pouvoir causal des états mentaux volitifs ne s'étend pas audelà des structures neuronales. Searle envisage un certain holisme dans le système cérébral à l'intérieur duquel il n'y a pas de frontière physiquement établie entre les traits. En ce sens, la causalité est un trait constitutif du système entier. Puisque le tout constitue un système physique, on peut faire une réduction quant à l'explication des conditions nécessaires de possibilité des phénomènes mentaux, c'est-à-dire une réduction épistémologique. Cependant, Searle refuse la réduction ontologique. À ses yeux, ce n'est pas parce que les éléments structurels sont des neurones que la totalité des traits du système est identique aux traits purement neuronaux. Il s'oppose ainsi à l'identité psychoneuronale. Cette position est-elle compatible avec son refus du dualisme des propriétés ? Dans son optique, il n'y a pas deux propriétés. Il y a un système de propriétés qui sont par nature des propriétés physiques. Pareilles propriétés peuvent exemplifier différents traits en même temps. Ce n'est pas parce que des traits mentaux surviennent à un certain point du système que le système entier laisse d'être un système physique.

D'autre part, Kim a tort de dire que l'approche de Searle n'apporte aucune solution au problème philosophique de la relation entre l'esprit et le corps. Car il faut reconnaître que Searle propose une solution originale: une approche naturaliste, mais non réductionniste. Les traits mentaux, en tant qu'éléments constitutifs du système neuronal, restent cependant un trait irréductible. D'un côté, Searle apporte une solution au problème philosophique. Il dit : "Votre douleur est causée par des séquences d'enchaînements neuronaux, mais c'est le cerveau qui est responsable de l'expérience de la douleur ${ }^{55}$. " Le système complet peut exemplifier ou co-exemplifier à la fois deux genres de traits. C'est une approche qui postule l'irréductibilité ontologique d'un certain type de traits (mentaux) des propriétés physiques. Elle reste cohérente, car elle ne postule pas deux types de propriétés. Cependant, il y a quelque inclination vers un certain dualisme quant aux traits des propriétés physiques.

Il faut admettre, par contre, que cette solution se limite au terrain de la philosophie. En fait, le problème concernant la vérification de l'hypothèse de la liberté de l'agent dans le cas volitif entraînera la détermination, par des recherches empiriques, du degré réel d'indéterminisme du système neurophysiologique. Son approche a le mérite de montrer que le problème de la causalité mentale ne concerne plus la surdétermination causale due à deux ensembles de propriétés ontologiquement rivales. Du même coup, il sauve l'esprit de la

55. J. R. Searle, Liberté et neuroscience, op. cit., p. 14. 
réduction ontologique. Dans sa vision, les traits constitutifs du système complet ne s'excluent pas mutuellement. L'esprit fait partie d'un tout unifié. Il est évident que Searle arrive par des analyses strictement philosophiques à des résultats dont la vérification dépasse le seuil de la philosophie. Il faudra compter sur le progrès des sciences en général, et de la neuroscience en particulier, pour évaluer la valeur de son approche. Les neurosciences doivent dorénavant répondre à la question: "Quels sont les processus neuronaux qui causent le vécu de nos expériences conscientes, intentionnelles et rationnelles, et comment ces expériences se réalisent-elles dans les structures du cerveau ? » par des preuves empiriques.

\section{Bibliographie}

Audi, Robert. The Cambridge Dictionary of Philosophy, (éd. Général), deuxième édition, Cambridge, Cambridge University Press, 1999.

Austin, J. L. How to Do Things with Words, Oxford, Clarendon Press, 1962.

Churchland, P. M. Neurophilosophy, Cambridge, MA, MIT Press, 1986.

Churchland, P. S. Scientific Realism and the Plasticity of Mind, Cambridge, Cambridge University Press, 1979.

Crane, Tim. "The Mental Causation Debate » in Proceedings of the Aristotelian Society Supplementary, volume LXIX, 1995, p. 211-236.

"Against Physicalism » in the Blackwell Companion to the Philosophy of Mind, Samuel Guttenplan, Oxford, Blackwell, 1995, p. 479-489.

Dennett. D. The Intentional Stance, Cambridge, MA, MIT Press, 1987.

- Consciousness Explained, Boston/New York/London, Little, Brown and Company, 1991.

Descartes, René. 1641. Méditations. Rééditées in Descartes: CEuvres et Lettres, textes présentés par A. Bridoux.

1649. Les passions de l'âme. Rééditées in Descartes: CEuvres et lettres, textes présentés par A. Bridoux, Paris, Gallimard, Bibliothèque de la Pléiade, 1953.

Dretske, Fred. Naturalizing the Mind, Cambridge, MA, MIT Press, 1995.

Fodor, Jerry. The Language of Thought, Cambridge, MA, Harvard University Press, 1975.

- "Making Mind Mater More» in A Theory of Content and Other Essays. Cambridge, MA, MIT Press, 1990.

Goldman, A. A Theory of Human Action. Princeton, New Jersey, Princeton University Press, 1976.

Heil, J. et A. Mele (dir.). Mental Causation, Oxford, Clarendon Press, 1995.

Kim, J. 1998. Mind in a Physical World. Cambridge, MA, The MIT Press.

. Physicalism, or Something Near Enough, Princeton and Oxford, Princeton University Press, 2005.

James, William. 1976a. «Does “consciousness" exist? » In Essays in radical empiricism. F. Burkhardt (dir.) p. 3-19. Cambridge, MA, Harvard University Press (première version publiée en 1904).

Mele, R. A. The Philosophy of Action, Oxford et New York, Oxford University Press, 1997.

Parsons, Terence. Events in The Semantics of English : A Study in Subatomic Semantics, Cambridge, MA, MIT Press, 1990. 
Putnam, Hilary. Mind, Language and Reality, vol. 2, Cambridge, Cambridge University Press, 1975.

Robb, D. "The Properties of Mental Causation », in Philosophical Quarterly 47, 1997, p. 178-95.

Russell, Bertrand. An Inquiry into Meaning and Truth, London, George Allen and Unwin, 1976.

Searle, John R. Intentionality, Cambridge, Cambridge University Press, 1983.

- Minds, Brains and Science, Cambridge, MA, Harvard University Press, 1984. - The Rediscovery of Mind, Cambridge, MA, MIT Press, 1992.

- Mind, Language and Society, Basic Books, 1998.

-. Rationality in Action, Cambridge, MA, MIT Press, 2001.

- "Consciousness, the Brain and the Connection Principle: A reply» in Philosophy and Phenomenological Research, vol. LV, n 1, mars 1995, p. 217 232.

- "Why I Am Not a Property Dualist ", Journal of Consciousness Studies, 9, no 12, 2002, pp. 57-64.

- Liberté et neurobiologie, édité et traduit par Patrick Savidan. Paris, éditions Grasset \& Fasquelle, 2004.

Searle, J. R. et D. Vanderveken. Foundations of Illocutionary Logic, Cambridge, Cambridge University Press, 1985.

S. P. Sitch. From Folk Psychology to Cognitive Science: The Case against Belief, Cambridge, MA, MIT Press, 1983.

Wittgenstein, Ludvig. 1922. Tractatus Logico-philosophicus. Trad. anglaise de D. F. Pears et B. F. McGuinness, London, Routledge \& Kegan Paul. 1961. Trad. française de Gilles Gaston Granger, Paris, Gallimard, 1993. 